\title{
Robust control chart for change point detection of process variance in the presence of disturbances
}

\begin{abstract}
A conventional control chart for detecting shifts in variance of a process is typically developed where in most circumstances the nominal value of variance is unknown and based upon one of the essential assumptions that the underlying distribution of the quality characteristic is normal. However, this is not always the case as it is fairly evident that the statistical estimates used for these charts are very sensitive to the occurrence of occasional outliers. This is for the reason that the robust control charts are put forward when the underlying normality assumption is not met, and served as a remedial measure to the problem of contamination in process data. Realizing that the existing approach, namely Biweight A pooled residuals method, appears to be resistance to localized disturbances but lack of efficiency when there are diffuse disturbances. To be concrete, diffuse disturbances are those that have equal change of being perturbed by any observation, while a localized disturbance will have effect on every member of a certain subsample or subsamples. Since the efficiency of estimators in the presence of disturbances can rely heavily upon whether the disturbances are distributed throughout the observations or concentrated in a few subsamples. Hence, to this end, in this paper we proposed a new robust MBAS control chart by means of subsample-based robust Modified Biweight A scale estimator in estimating the process standard deviation. It has strong resistance to both localized and diffuse disturbances as well as high efficiency when no disturbances are present. The performance of the proposed robust chart was evaluated based on some decision criteria through Monte Carlo simulation study.
\end{abstract}

Keyword: Change point detection; Control chart; Outlier; Robust estimation; Statistical process control 